\title{
ON THE MODULAR GROUP RING OF A p-GROUP
}

\author{
DONALD B. COLEMAN
}

Let $G$ be a finite $p$-group, $p$ a prime, and let $F$ be a field of characteristic p. $F(G)$ will denote the group ring of $G$ over $F$. In the first section of this note, two elementary results are given that concern $G$ as a subgroup of the group of units of $F(G)$. In the second section the center of $F(G)$ is considered.

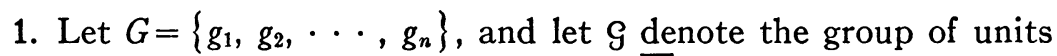
of $F(G) . g$ consists of those elements $x=\sum_{1}^{n} \alpha_{i} g_{i}$ in $F(G)$ such that $c(x)=\sum_{1}^{n} \alpha_{i} \neq 0$. Moreover, $\mathcal{S}=\mathcal{G}^{*} \times F^{*}$, where $\mathcal{G}^{*}=\{x: x \in F(G)$, $c(x)=1\}$ and $F^{*}$ is the multiplicative group of nonzero members of $F$. Note that $\mathcal{G}^{*}=\mathfrak{N}+1$, where $\mathscr{N}$ is the radical of $F(G)$. (See $[4, \mathrm{p}$. 175].) $Z$ will denote the center of $G$.

Theorem 1. Let $N$ be the normalizer of $G$ in $g$. Then $N=G \mathrm{Z}$.

Proof. Clearly $G z \subset N$.

Let $x=\sum \alpha_{i} g_{i} \in N$. For each $g_{j} \in G$, let $g_{k}(k=k(j))$ be such that $x g_{k}=g_{j} x$. For each $j=1,2, \cdots, n$, let $\gamma_{j}$ be the permutation of $G$ defined by

$$
g^{\gamma_{j}}=g_{j} g g_{k}^{-1} .
$$

$\gamma_{j}=\tau_{j} \beta_{j}=\beta_{j} \tau_{j}$, where $\tau_{j}: g \rightarrow g_{j} g$ and $\beta_{j}: g \rightarrow g g_{k}^{-1}$. The orders of $\tau_{j}$ and $\beta_{j}$ are powers of $p$. Hence $\gamma_{j}$ has order a power of $p$. Each member of the group $S$ generated by $\gamma_{1}, \gamma_{2}, \cdots, \gamma_{n}$ has a similar form, so that $S$ is a $p$-group of permutations of $G$. Moreover $x g_{k}=g_{i} x$ if and only if $\alpha_{r}=\alpha_{s}$ whenever $g_{s}^{\gamma_{j}}=g_{r}$. Thus the coefficients of $x$ must agree on the transitivity classes of $S$. Since $S$ is a $p$-group, these classes consist of either 1 or a power of $p$ elements each. Since $F$ has characteristic $p$, and since $c(x) \neq 0$, it follows that some transitivity class of $S$ must consist of a single element. Hence $S$ has a fixed point, say $g$.

Then $g=g_{j} g g_{k}^{-1}$ for each $j=1,2, \cdots, n$, so that $g^{-1} g_{j} g=g_{k}$ $=x^{-1} g_{j} x(j=1,2, \cdots, n)$. Hence $x \equiv g(\bmod C(G))$, where $C(G)$ denotes the centralizer of $G$ in $\mathcal{G}$; and since $C(G)=\mathcal{Z}$, it follows that $x \in G Z$. This completes the proof.

Let $(x, y)=x^{-1} y^{-1} x y(x, y \in \mathcal{G})$.

Corollary. Let $x \in \mathcal{G}, g_{j} \in G$. If $\left(g_{j}, x\right) \in G$, then $\left(g_{j}, x\right)=\left(g_{j}, g\right)$ for some $g \in G$.

Received by the editors February 27, 1963. 
Proof. The proof follows that of Theorem 1, except here we only need to use the fact that $\gamma_{j}$ has a fixed point.

Corollary. If $C$ is the conjugate class in 9 containing the element $g \in G$, then $C \cap G$ is the conjugate class in $G$ that contains $g$.

For a group $H$, let $H^{\prime}$ denote the commutator subgroup of $H$.

THEOREM 2. $G \cap g^{\prime}=G^{\prime}$.

Proof. Let $I\left(G^{\prime}\right)$ denote the ideal in $F(G)$ generated by elements of the form $g-1, g \in G^{\prime}$. Let $X=\left\{x+1: x \in I\left(G^{\prime}\right)\right\}$. It is easily verified that $X$ is a normal subgroup of $\mathcal{G}$ (in fact, of $\mathcal{G}^{*}$ ). According to $\left[2\right.$, p. 36], $X \cap G=G^{\prime}$. The quotient algebra $F(G) / I\left(G^{\prime}\right)$ is commutative $\left[1\right.$, p. 2]. Thus for each $x, y \in \mathcal{G},(x, y)-1 \in I\left(G^{\prime}\right)$, so that $(x, y) \in X$; i.e., $\mathcal{G}^{\prime} \subset X$. Hence $\mathcal{G}^{\prime} \cap G \subset G^{\prime}$. Obviously $G^{\prime} \subset \mathcal{G}^{\prime} \cap G$. This completes the proof.

2. Let $C_{1}, C_{2}, \cdots, C_{t}$ denote the noncentral conjugate classes of $G$; for each $i=1,2, \cdots, t$, let $K_{i}=\sum_{x \in C_{i}} x$. It is well known that if $Z=\left\{z_{1}, z_{2}, \cdots, z_{m}\right\}$ is the center of $G$, then the elements $z_{1}, \cdots, z_{m}$, $K_{1}, \cdots, K_{t}$ form a basis for the center $Z F(G)$ of $F(G)$.

Theorem 3. (1) $K_{i} K_{j}=\sum_{k} c_{i j k} K_{k}$, with $c_{i j k} \in G F(p) ; i, j=1,2$, $\cdots, t$. Thus the sub-(vector) space of $F(G)$ with basis $K_{1}, \cdots, K_{t}$ is an ideal in $Z F(G)$.

(2) Suppose that $G$ satisfies the condition that $\left(a^{p}, b\right)=1$ if and only if $\left(a, b^{p}\right)=1 ; a, b \in G$. Then $K_{i}^{p}=0 ; i=1,2, \cdots, t$.

Proof. (1) Suppose that $K_{i} K_{j}=\sum_{1}^{m} \alpha_{r} z_{r}+\sum_{1}^{t} \beta_{s} K_{8} ; \alpha_{r}, \beta_{s}$ nonnegative integers.

Let $g_{i}$ and $g_{j}$ be members of $C_{i}$ and $C_{j}$, respectively. Then for $g_{i}^{x}$ and $g_{j}^{\nu}$ in $C_{i}$ and $C_{j}$, it follows that

$$
{\stackrel{x}{g_{i}} g_{j}^{y}}^{y}=z_{r} \in Z
$$

if and only if $g_{i}^{x y^{-1}}=g_{j}^{-1} z_{r}$. (Here $a^{b}=b^{-1} a b$.)

If for fixed $x$ and $r$, there is some $y \in G$ such that $\left(^{*}\right)$ holds, then

$$
g_{i}^{x}=\left(g_{j}^{-1}\right)^{y} \cdot z_{r}=\left(g_{j}^{-1} z_{r}\right)^{\nu},
$$

so that $\left(^{*}\right)$ holds for exactly one $y$ modulo $C\left(g_{j}^{-1} z_{r}\right)=C\left(g_{j}\right)$.

Thus if $C_{i}=\left\{g_{i}^{x_{q}}: q=1,2, \cdots, u\right\}$ and if $C_{j}=\left\{g_{j}^{y_{s}}: s=1,2, \cdots, v\right\}$, and if $g_{i}$ and $g_{j}^{-1} z_{r}$ are conjugate, then for each $x_{q}$, there is a unique $y_{*}(1 \leqq s \leqq v)$ such that 


$$
g_{i}^{x_{q}} g_{j}^{y_{s}}=z_{r} .
$$

Hence $\alpha_{r}=u \equiv 0(\bmod p)$. If $g_{i}$ and $g_{j}^{-1} z_{r}$ are not conjugate, then $z_{r}$ does not occur in the group product $C_{i} C_{j}$, so that $\alpha_{r}=0$.

(2) Let $K=K_{i}$, with $C=C_{i}$ a class containing an element $g$.

Case 1. Assume that $C$ is a commutative set. Choose a subgroup $H$ of $G$ containing $C(g)$ such that $|H: C(g)|=p$. Then let $D=\left\{g, g^{x}, \cdots, g^{x^{p-1}}\right\}$ be the conjugate class in $H$ that contains $g$, with $g^{x^{p}}=g$; i.e., $\left(g, x^{p}\right)=1$. Then by hypothesis, $\left(g^{p}, x\right)=1$, so that for $L=\sum_{y \in D} y$, we have

$$
L^{p}=g^{p}+x^{-1} g^{p} x+\cdots+x^{-(p-1)} g^{p} x^{p-1}=p g^{p}=0 .
$$

Let $C=D_{1} \cup D_{2} \cup \ldots \cup D_{q}$, with $D_{i} \cap D_{j}=\varnothing$ for $i \neq j$, and with each $D_{i}$ conjugate to $D$. Then for $L_{i}=\sum_{y \in D_{i}} y$, we have

$$
K=L_{1}+L_{2}+\cdots+L_{q}
$$

so that

$$
K^{p}=L_{1}^{p}+L_{2}^{p}+\cdots+L_{q}^{p}=0 .
$$

Case 2. Assume that $g$ fails to commute with one of its conjugates. The proof is by induction on $n=|G|$, the order of $G$.

Since $C \subset g G^{\prime} \subset g \Phi$, where $\Phi$ is the Frattini subgroup of $G$, and since $G$ is not cyclic, we see that the subgroup $W$ of $G$ generated by $C$ is proper. Let $C$ decompose into conjugate classes $D_{1}, D_{2}, \cdots, D_{w}$ in $W$. As before, let $L_{i}$ denote the sum of elements in $D_{i}$. If $D_{i}$ is commutative, then $L_{i}^{p}=0$ by Case 1 . If not, since $D_{i}$ is a conjugate class in a $p$-group of order less than $n$ (which satisfies the standing hypothesis of (2)), then $L_{i}^{p}=0$ by induction. Hence $K^{p}=L_{1}^{p}+L_{2}^{p}+\cdots+L_{w}^{p}$ $=0$. This completes the proof.

Note that a regular $p$-group satisfies the condition of (2) [3, p. 185]. If $C$ is a commutative class, then $K^{p} \neq 0$ if and only if $C(g)=C\left(g^{p}\right)$; in this case $K^{p}=L$, where $L$ is the sum of the elements in the class containing $g^{p}$.

The following theorem is due to Deskins [2, p. 39]. An alternate proof will be given here and it will be followed by a slightly more general theorem.

Theorem 4 (Deskins). If $G$ and $H$ are finite Abelian p-groups, and if $F(G) \cong F(H)$ for some field $F$ of characteristic $p$, then $G \cong H$.

Proof. For any set $A$ of group ring elements, let $A^{p}=\left\{a^{p}: a \in A\right\}$. This proof of the theorem is by induction on $n=|G|=|H|$. 
Under any isomorphism of $A=F(G)$ onto $B=F(H)$, it is clear that $A^{p}$ maps onto $B^{p}$. But $A^{p}=F^{p}\left(G^{p}\right)$ and $B^{p}=F^{p}\left(H^{p}\right)$ where $F^{p}$ is the field of $p$ th powers of members of $F$. Hence by induction, since $\left|G^{p}\right|=\left|H^{p}\right|<n$, we have that $G^{p} \cong H^{p}$. For finite Abelian p-groups $G$ and $H$, the two conditions (i) $G^{p} \cong H^{p}$ and (ii) $|G|=|H|$ are enough to ensure that $G$ and $H$ are isomorphic.

THEOREM 5. Let $G$ and $H$ be finite p-groups satisfying the condition of Theorem 3(2). If $Z F(G) \cong Z F(H)$, then $Z_{1}^{p} \cong Z_{2}^{p}$, where $Z_{1}$ and $Z_{2}$ denote the centers of $G$ and $H$, respectively. Thus in this case, if $Z_{1}$ and $Z_{2}$ have the same order, then $Z_{1} \cong Z_{2}$.

Proof. By Theorem 3(2),

$$
[Z F(G)]^{p}=\left[F\left(Z_{1}\right)\right]^{p}=F^{p}\left(Z_{1}^{p}\right)
$$

and

$$
[Z F(H)]^{p}=\left[F\left(Z_{2}\right)\right]^{p}=F^{p}\left(Z_{2}^{p}\right) .
$$

Thus if $Z F(G) \cong Z F(H)$, it follows that $F^{p}\left(Z_{1}^{p}\right) \cong F^{p}\left(Z_{2}^{p}\right)$. Hence by Theorem $4, Z_{1}^{p} \cong Z_{2}^{p}$.

In $[2$, p. 39] it is stated that Theorem 4 fails for arbitrary $p$ groups, and the quaternion group and the dihedral group of order 8 are said to have isomorphic group rings over $G F(2)$. This is not, however, the case. For in the group ring of the quaternion group all units of order 2 are central. This is not true, of course, for the dihedral group.

\section{BIBLIOGRAPHY}

1. D. B. Coleman, Finite groups with isomorphic group algebras, Trans. Amer. Math. Soc. 105 (1962), 1-8.

2. W. E. Deskins, Finite Abelian groups with isomorphic group algebras, Duke Math. J. 23 (1956), 35-40.

3. M. Hall, The theory of groups, Macmillan, New York, 1959.

4. S. A. Jennings, The structure of the group ring of a p-group over a modular field, Trans. Amer. Math. Soc. 50 (1941), 175-185.

VANDERBILT UNIVERSITY 